\title{
Induction stage-dependent expression of vascular endothelial growth factor and aquaporin-1 in diethylstilbestrol-treated rat pituitary
}

\author{
W. Zhao, ${ }^{1, *}$ H. Shen, ${ }^{2, *}$ F. Yuan, ${ }^{1}$ G. Li, ${ }^{1}$ Y. Sun, ${ }^{1}$ Z. Shi, ${ }^{1}$ Y. Zhang, ${ }^{1}$ Z. Wang ${ }^{1}$ \\ ${ }^{1}$ Beijing Neurosurgical Institute, Capital Medical University, Beijing; ${ }^{2 B e i j i n g ~ T i a n t a n ~ H o s p i t a l, ~ C a p i t a l ~}$ \\ Medical University, Beijing China; *the two authors contributed equally to the work
}

\begin{abstract}
(C2009 European Journal of Histochemistry
The anterior pituitary gland undergoes tumourigenic changes in response to oestrogen treatment in several breeds of rats. We administered diethylstilbestrol (DES) to female Wistar rats and assessed whether the expression of vascular endothelial growth factor (VEGF) and aquaporin-1 (AQP-1) was altered at different time points following DES administration. In vivo magnetic resonance imaging (MRI) scans showed that the mass index corresponding to the mid-sagittal area of DES-treated pituitary was significantly higher than the vehicle-controlled pituitary $(p<0.01)$ at three specific time points, accompanied by a significant reduction in body weight. Haematoxylin and eosin (HE) staining and immunohistochemical analysis demonstrated that during early stages of induction, DES increased cell proliferation and sprouting of endothelial cells, and VEGF expression transitioned from a vessel-surrounding pattern to a diffuse pattern. During later stages, angiogenesis was predominant, and VEGF expression decreased. In contrast to the early abundant expression of VEGF, endothelial expression of AQP1 increased during later stages. Our data indicated a dynamic scenario of biological alterations in DES-treated pituitary tissue, in which VEGF and AQP-1 exert their functions at different stages of induction, and we provide novel insights into understanding oestrogen-related tumourigenesis in the anterior pituitary gland.
\end{abstract}

Key words: diethylstilbestrol, angiogenesis, vascular endothelial growth factor, aquaporin-1.

Correspondence: Fang Yuan, Guilin Li,

Department of Pathophysiology, Beijing Neurosurgical Institute, Capital Medical University, Beijing 100050, China Tel. +86.1067096750.

E-mail: florayuan@vip.sina.com and liguilin40@hotmail.com

Paper accepted on February 19, 2009

European Journal of Histochemistry

2009; vol. 53 issue 1 (January-March): 53-60
$\mathrm{M}$ orphological changes of the pituitary tumour induced by oestrogen in vitro predominantly include increased cell proliferation and angiogenesis. In contrast to the large number of studies assessing the effects of oestrogen on mature pituitary tumour tissue, the morphological changes and differential expression of relevant molecules at specific stages of induction have not been thoroughly investigated.

Angiogenesis, a condition of neovascularisation that arises from existing vasculature, plays pivotal roles in foetal development, injury repair, and reproductive cycle regulation. Additionally, it is a prerequisite for the induction of tumour growth and invasion (Gimbrone et al. 1972, Gimbrone et al. 1973, Folkman et al. 1990). Angiogenesis critically contributes to tumourigenesis and invasion of pituitary tumours, and it affects the response of these tumours to therapeutic intervention (Goth et al. 2003). High-density vascularisation is associated with increased invasion potential in human mammary tumours, as well as bladder and gastric neoplasias; thus, it contributes to a poorer prognosis and low survival rates (Weidner et al. 1991, Weidner et al. 1992, Maeda et al. 1995, Bochner et al. 1995). Angiogenesis results from signalling mediated by multiple factors (Guinebretiere, 2005); particularly vascular endothelial growth factor (VEGF), which in turn mediates the biological effects of other growth factors. Aquaporins (AQPs) are a family of proteins that shuttle water across the cellular membrane, and a subset of these proteins can counteract glioma-associated lactic acidosis by clearing glycerol and lactate from the extracellular space (Warth, 2007). AQP-1 mRNA and protein are expressed at very low levels in rat brain primary microvascular endothelial cells, and its expression increases with passaging (Dolman, 2005). AQP-1 has also been detected in the pituitary gland and is expressed in vascular endothelial 
cells of the adenohypophysis and neurohypophysis (Kuwahara, 2007). However, expression of AQP-1 in pituitary in response to oestrogen is still not clear.

In the current study, DES was intraperitoneally administered to rats to induce chronic tumourigenesis in the anterior pituitary gland.

Magnetic resonance imaging (MRI) and haematoxylin and eosin (HE) staining were applied to monitor in vivo tumour growth and morphological changes. Electron microscopy was utilised to assess the final ultrastructural attributes of the pituitary gland. Immunohistochemistry was used to evaluate the expression of VEGF and AQP-1 and their localisation at specific stages following DES administration.

\section{Materials and Methods}

A total of 60 female Wistar rats, 3 weeks old and weighing 70-80 g, were used. The animals were housed with free access to tap water and standard pellet food. They were kept at a controlled temperature $\left(24 \pm 1^{\circ} \mathrm{C}\right)$ and humidity $(55 \pm 5 \%)$, and a 12 hour day-night cycle (10 p.m.-10 a.m.) was maintained. The induction was based on a previously published method (Zhao et al. 2007). Rats were randomly allocated into two groups for each time point. In the vehicle-controlled group, rats were injected intraperitoneally with sunflower seed oil ( $1 \mathrm{~mL} / \mathrm{kg}$, twice a week) for 12 weeks, whereas animals in the DES group were administered DES intraperitoneally (5 $\mathrm{mg} / \mathrm{kg}$, twice a week) for 12 weeks. The procedures undertaken were in strict compliance with the guidelines on the care and use of laboratory animals at our institution. DES was purchased from Sigma Chemical Co. (St Louis, MO, USA). Anti-VEGF antibody was purchased from Boster Biotech (Wuhan, China), and anti-AQP-1 antibody was purchased from Chemicon (Temecula, CA, USA). The DES injection was prepared by dissolving DES powder in sunflower seed oil to obtain a final concentration of $5 \mathrm{mg} / \mathrm{mL}$.

\section{MRI scanning}

At week 4, week 8, and week 12, three rats were randomly selected from each group for MRI scanning. The $T_{2}$ WI mid-sagittal scanning technique was used to monitor the changes in the pituitary gland at each time point. Intraperitoneal administration of hydration chloraldehyde at $30 \mathrm{mg} / 100 \mathrm{~g}$ of body weight was applied to minimise animal movement during scanning. The rats were placed in a specifically designed saddle-coil probe, and a GEMSL×3T superconductive MR (General Electric, Fremont, CA) medical imaging instrument was used to obtain a mid-sagittal $T_{2} W I$ image with a TR of $3100 \mathrm{~ms}$ and a TE of 115 ms. Data collection matrix sizes were $320 \times 288$ for $T_{2} W I$. The mid-sagittal section was defined as the in-line position of the olfactory bulb, corpus callosum, cerebellum, and brain stem. The software Image Tool II was used to obtain the mid-sagittal area indexed by the number of pixels within each rat pituitary gland.

\section{Morphological methods}

At each time point, rodents were weighed and anaesthetised by hydration chloraldehyde at 30 $\mathrm{mg} / 100 \mathrm{~g}$ body weight and transcardially perfused with a $40 \mathrm{~g} / \mathrm{L}$ solution of paraformaldehyde diluted in PBS $(0.01 \mathrm{M}, \mathrm{pH} 7.4)$. The tissues were embedded in paraffin and sectioned at $4 \mu \mathrm{m}$ for HE staining and immunohistochemistry.

Immunohistochemical staining was applied to demonstrate the expression and localisation of VEGF and AQP-1. In brief, deparaffinised sections were rehydrated through a graded series of ethanol to phosphate buffered saline (PBS). Antigen retrieval was performed, and endogenous peroxidase clearance was performed by incubation in 3\% $\mathrm{H}_{2} \mathrm{O}_{2}$. Then sections were blocked with $10 \%$ normal goat serum for 30 minutes. Afterwards, these sections were individually incubated with anti-VEGF and anti-AQP-1 antibodies (diluted at 1:50) at $4^{\circ} \mathrm{C}$ overnight. The antigen-antibody complexes were visualised using the avidin-biotin-peroxidase complex $(A B C)$ method. Counterstaining was performed with Mayer's haematoxylin.

\section{Transmission electron microscopy}

After induction, one rat from the vehicle control group and two rats from the DES group were randomly selected for transmission electron microscopic analysis. For electron microscopy, pituitary tissues were fixed in $2 \%$ glutaraldehyde in $0.1 \mathrm{M}$ PBS ( $\mathrm{pH} 7.4$ ) and then subjected to postfixation in $2 \%$ osmium tetroxide. The tissues were embedded in Araldite, and the ultra thin sections were examined using electron microscopy (Philips EM208).

\section{Statistical analysis}

Results were expressed as means \pm standard devi- 
ation (SD), and one-way ANOVA analysis was utilised to compare the differences between the two groups in terms of body weight, mid-sagittal area, with post hoc testing using the Bonferroni procedure. $P$ values less than 0.05 were considered significant.

\section{Results}

\section{Body weight}

Fur loss in the DES group was initially observed as early as 2 weeks after the initiation of DES treatment and was sustained throughout the remainder of the study (data not shown). At weeks 4,8 , and 12 , the body weights in the vehicle control group were $189.6 \pm 15.6 \mathrm{~g}, 256.4 \pm 27.0 \mathrm{~g}$, and $244.5 \pm 23.8 \mathrm{~g}$, respectively, whereas in the DES group they were $164 \pm 11.4 \mathrm{~g}, 209.2 \pm 13.5 \mathrm{~g}$ and $208.4 \pm 21.0 \mathrm{~g}$, respectively. Therefore, body weight was significantly reduced in the DES group at each time point $(n=10$ in each group, and $p<0.05)$ compared with the vehicle-controlled group.

\section{MRI changes}

At three time points, $T_{2}$ WI scanning of the pituitary gland in all tested rats in the vehicle-controlled group indicated a typical triangular pattern with sharp margins and uniform image signal intensity lighter than that of the surrounding brain tissue. The peripheral meninges and cerebral spinal fluid (CSF) were also continuous. No abnormal changes in the shape were observed in the vehicle-controlled group throughout the course of the study (Figure $1 \mathrm{Al}-3$ ). At week 4 , the pituitary gland in the DES-treated rats started to enlarge, losing its typical triangular shape, and the dura mater showed heterogeneous signals under T2WI, with a blurred subarachnoid space (Figure $1 \mathrm{Bl}$ ). At week 8, a discontinuous dura mater, further enlarged pituitary gland, and heterogeneous intrahypophyseal signal intensity were observed (Figure 1 B2). At week 12, the pituitary gland crept over the sella turcica in a hillock-like shape. The continuity of the dura mater was severely destroyed, and the enlarged pituitary gland expanded into the subarachnoid space, resulting in a malformed pituitary gland in the DES-treated rats (Figure 1 B3). MRI scanning has been proved to be a practical method for monitoring the growth and morphological alterations of the pituitary gland induced by oestrogen treatment, and the mid-sagittal area closely correlates with the mass of the pituitary gland. During the progression of these studies, the pituitary gland in the control group exhibited slow growth; the indexed number of pixels was $67.0 \pm 4.6$, $75.7 \pm 7.6$, and $88.3 \pm 3.2$ at weeks 4, 8, and 12, respectively. In contrast, the mid-sagittal areas of the pituitary tissue in the DES-treated group were $131.0 \pm 11.5,160.7 \pm 11.7$ and $315.67 \pm 11.6$, respectively, which was significantly higher than in the control group ( $n=3$ in each group and $p<0.01$ in comparison to the controls) (Figure $1 \mathrm{C}$ ).
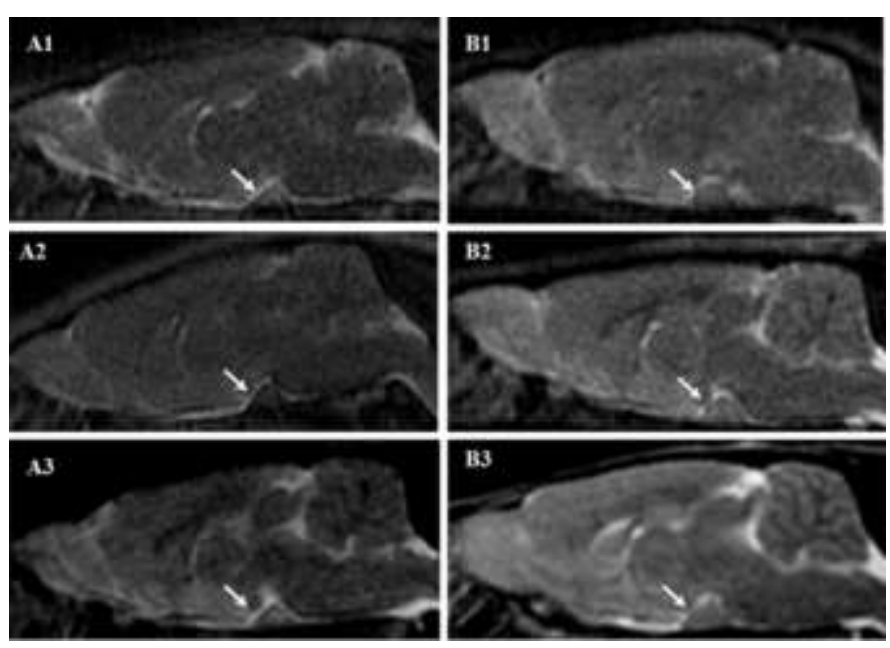

C

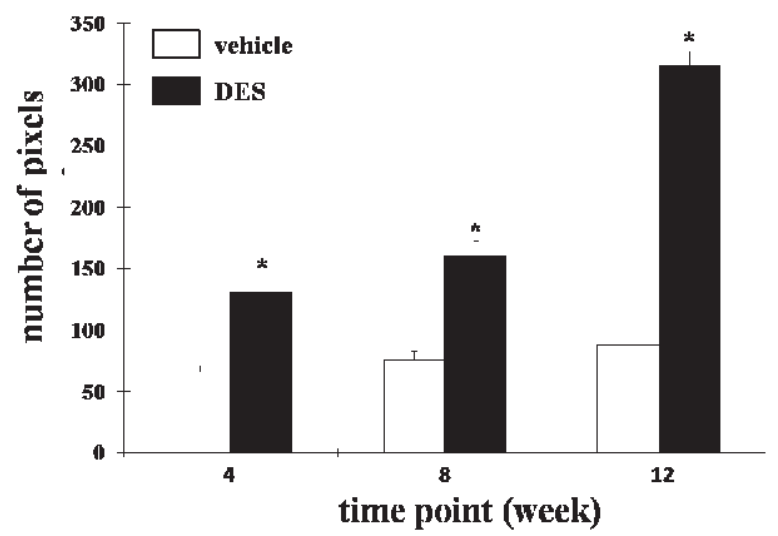

Figure 1. Representative pictures showing the rat pituitary gland under mid-sagittal $\mathrm{T}_{2} \mathrm{WI}$. In vivo MRI scanning showed that DES induced gradual enlargement of the pituitary gland (indicated by the arrow) at 4, 8, and 12 weeks (B 1-3), in contrast to the lack of change in the vehicle-treated pituitary (A1-3). As shown in C, long-term treatment of DES resulted in significantly larger mid-sagittal areas of the pituitary gland compared with the vehicle controls $\left({ }^{*} p<0.01\right.$, compared with controls, respectively). 


\section{Histology and immunohistochemistry}

Initially after the administration of DES, localised cell proliferation was observed (Figure $2 \mathrm{~A}$ ), and at 8 weeks, there was further spreading of proliferative cells. A typical characteristic of this stage is the sprouting of spindle-like endothelial cells lining the proliferative cells and the formation of large cordlike structures (Figure 2B). At this stage, no vascularised lumens were apparent in the area of proliferation. At week 12, vascular lakes were predominant in the anterior pituitary gland (Figure 2C); however, there was still a discernable borderline between

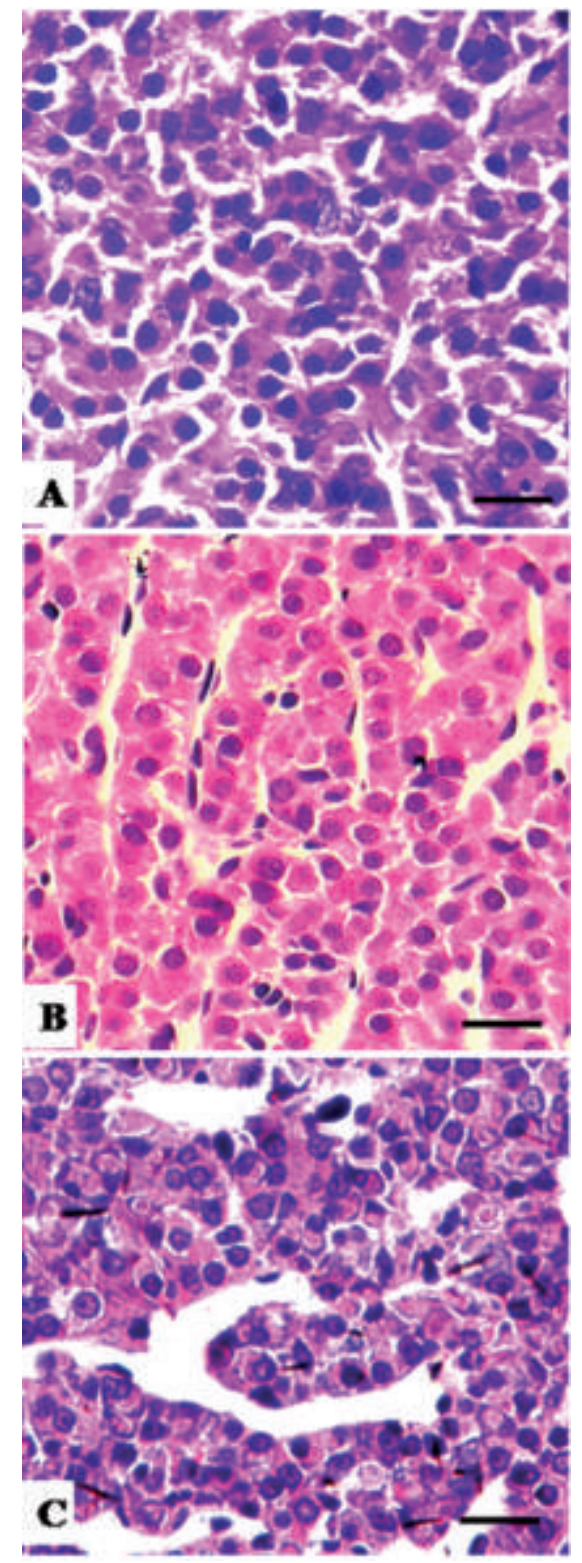

Figure 2. HE staining showing the morphological changes of the pituitary tissues subjected to DES treatment at week 4 (A), week 8 (B), and week 12 (C), respectively. (Scale bars, $20 \mu \mathrm{m}$ ). areas with and without angiogenesis. Notably, in the area without angiogenesis, cells with condensed nuclei were visible, which was rarely observed in vascular lake-enriched areas (data not shown). Based on the dynamic alterations observed from HE staining of DES-treated rat pituitary tissue, we found that angiogenesis characterised by vascular lake formation was a subsequent event to increased cell proliferation during pituitary tumourigenesis. We hypothesised that different factors may function during specific stages, so we investigated the expression of VEGF, a widely accepted angiogenic growth factor, and aquaporin-1 (AQP-1), a potential angiogenic stimulator, in response to DES administration over a 12-week time course. Throughout tumour induction and progression, expression of VEGF was weak and sparse in the vehicle-controlled group (data not shown). We could not detect the expression of VEGF in the endothelial cells in either group at any time point. Initially, in the DES-treated group, cytoplasmic VEGF-positive cells were mainly localised to the areas surrounding vessels (Figure 3A). At week 8, a diffuse expression pattern of VEGF was observed, whereas a subset of cells exhibited a perinuclear pattern (Figure 3B). When angiogenesis was complete at week 12 , the intensity of VEGF expression was reduced (Figure 3C); however, VEGF-positive cells were still prevalent in angiogenic areas in comparison to less angiogenic areas, resulting in sharp separation of the two niches (Figure 3D). Prior to the completion of angiogenesis, we could not recognise differential expression of AQP-1 between the two groups (data not shown), but by week 12, expression of AQP-1 was enhanced in the endothelial cells that lined the vascular walls (Figure 4). In contrast, there was no apparent expression of AQP-1 in proliferating cells.

\section{Transmission electron microscopy}

The intracellular membrane localisation of a secretory particle was detected in DES-treated pituitary tissue at week 12 (Figure 5A). The rough endoplasmic reticulum (RER) was well developed into a concentric-circled structure, a typical characteristic for the development of prolactinoma (Figure 5B). Concurrently, secretory granules with diameters ranging from $100 \mathrm{~nm}$ to $300 \mathrm{~nm}$ accumulated in areas adjacent to the vascular wall (Figure $5 \mathrm{C}$ ). The vascular wall surrounding neovascularised vessels was thin and poorly developed and had endothelial cells attached (Figure 5D). 

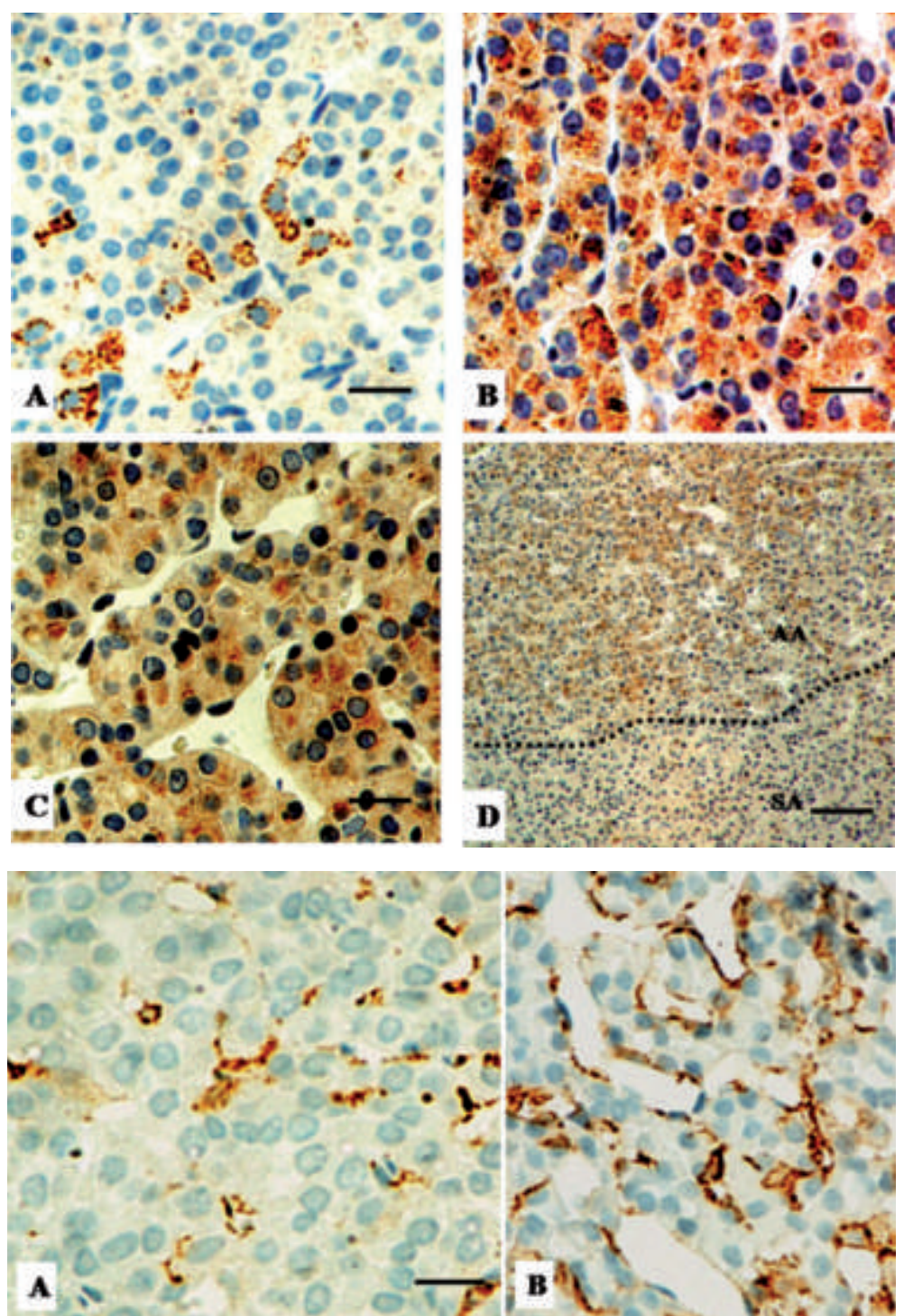

Figure 4. Ilmmunostaining for the expression of AQP1 in the vehicle-controlled group (A) and DES group (B) at week 12. A weak signal of AQP-1 expression was detected in the normal hypophyseal vascular walls (A), and DES-induced expression of AQP-1 was exclusively localised to the endothelial cells of neovascularised vessels (B) (scale bars: $A$ and $B, 20 \mu \mathrm{m}$ ).
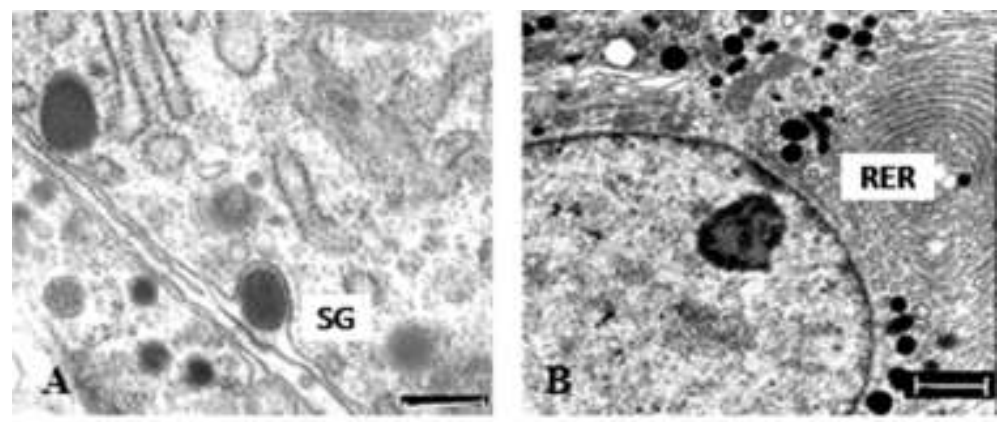

Figure 5. Representative image showing the ultrastructural results of the anterior pituitary gland treated with DES (SG: secretion granule; RER: rough endoplasmic reticulum; EC: endothelial cell) (scale bars: A, $300 \mathrm{~nm}$; B, C, and D, $1 \mu \mathrm{m})$.

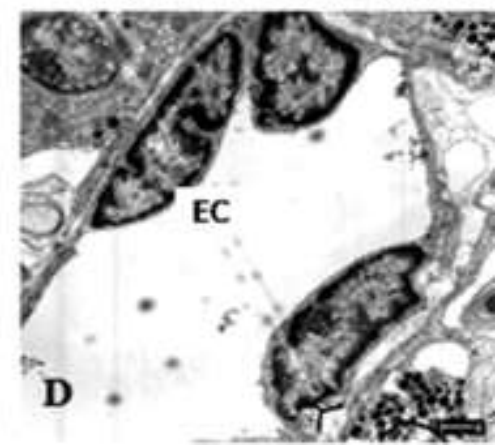




\section{Discussion}

Oestrogen has proven to be an efficient tumourigenic inducer for the pituitary gland, and several events contribute to this process. Intraperitoneal injection of oestrogen provided chronic tumourigenesis induction with real time dose modulation according to body weight. This method facilitates monitoring of the dynamic changes in neoplastic growth in vivo through MRI and immunohistochemistry, enabling the identification of morphological and expression alterations.

The mid-sagittal accumulated pixel number under T2WI highly correlated with pituitary body mass, suggesting that it is a high fidelity tool to evaluate rat pituitary mass in vivo (Rudin, 1988). Van Nesselrooij (van Nesselrooij, 1992) found that MRI could detect enlarged pituitary glands in rats as early as 16 days after treatment with estradiol-17. Our results showed that DES accumulation correlated with increased mid-sagittal area, which reached its peak at week 12, suggesting increased pituitary volume and mass.

MRI investigation also detected a blurred and discontinuous CSF signal representing the narrowing of the interstitial space between the pituitary tissue and dura mater at week 12 . This suggests that enlargement of pituitary tissue may oppress adjacent structures such as the dura mater and pons.

In previous studies, non-canonical proliferation of pituitary cells that occurred with the emergence of blood-enriched space was defined as a pituitary tumour (Satoh, 1997). In our study, in vivo MRI test and HE staining provided detailed information on the dynamic morphological changes that occurred during tumourigenesis of the pituitary gland. The pituitary cell proliferation transitioned from a local pattern to a diffuse pattern with the development and completion of neovascularisation. Angiogenesis was apparent at week 12, which may have contributed to the rapid increase in volume of the pituitary gland, as confirmed by MRI at week 12 .

The dynamic morphological changes led us to hypothesise that specific growth factors may be expressed in a distinct pattern during pituitary tumourigenesis. To assess this, we performed immunostaining for VEGF, a widely accepted key regulator of angiogenesis, and $\mathrm{AQP}-1$, a candidate stimulator of angiogenesis, proliferation and metastasis. VEGF, a homodimeric heparin-binding affinity protein with a molecular weight of 45 $\mathrm{kDa}$, was initially extracted from bovine pituitary follicular cells by Gospodarowicz et al. (Gospodarowicz, 1989). VEGF interacts with its receptor to signal in a paracrine fashion, specifically exerting mitogenic effects on nearby endothelial cells. Additionally, VEGF can increase the osmotic ability of the vascular endothelia, leading to the extravasation of serum proteins, fibre sedimentation, damage to the blood-brainbarrier due to enhanced endothelial proliferation. VEGF expression was detected under conditions of ischaemia, anoxia, high blood sugar levels, and hormone exposure. VEGF expression has been observed in rat and human growth hormone $(\mathrm{GH})$ pituitary tumours, prolactinoma, and adrenocorticotropic hormone (ACTH) adenoma, which can be stimulated by transformation factors including oestrogen and inhibited by dexamethasone (Loyhrer, 2001). In the normal pituitary gland, expression of VEGF was mainly restricted to endothelial cells, but here, we detected expression of VEGF in proliferative pituitary cells, but not in endothelial cells. This is consistent with Pan's report that VEGF was strongly expressed in pituitary adenomas and correlated with the tumour grade (Pan, 2003). Banerjeei et al. (Banerjeei, 2000) determined that methoxyestradiol could downregulate VEGF expression and potentially inhibit tumour growth by reducing expression of the pituitary tumour transforming gene (PTTG) (McCabe, 2002; Heaney, 2002).

Expression of VEGF was initially limited to areas surrounding the vessels. We hypothesise that these cells initially sensitise and react to the stimulation of DES by upregulating the expression of VEGF because they are in direct contact with the vascular wall. At week 8, VEGF was expressed in a diffuse pattern due to continuous stimulation of DES. Notably, perinuclear localisation of VEGF was noted in a subset of proliferative cells. The nuclear localisation of VEGF was additionally confirmed by immune electron microscopy, which indicated that VEGF associates with the nucleolus and euchromatin (Jorge, 2005). The nuclear translocation of VEGF endows the tumour cells with stronger proliferative ability and increased transcription of protumourigenic and angiogenic factors. During the final stages of induction, the expression of VEGF 
was sharply reduced, but the differential expression of VEGF prevailed between areas where angiogenesis was present and absent. Although the pituitary VEGF level correlates well with mass (Cracchiolo, 2002), high serum levels of VEGF are not a prerequisite, suggesting that additional factors are involved in this process.

We present the first study examining the expression of aquaporin-1 in response to DES stimulation, and we evaluate its potential role as a stimulator of angiogenesis and executor of metastasis. Although it has been reported that transfection of $A Q P-1$ into the pituitary endocrine cell line AtT20 affects dense core secretory granule (DCSG) biogenesis and normal levels of hormonal secretion (Arnaoutova, 2008), we failed to detect the expression of AQP-1 in the pituitary cells in both groups at each time point. In contrast, at week 12, AQP-1 was strongly expressed in the vascular endothelial cells, suggesting that AQP-1 may function as an executor of angiogenesis and possibly indirectly affect cell proliferation, tumour cell migration, and invasion. AQP1-knockout mice exhibited reduced tumour angiogenesis, suggesting that AQP-1 facilitates cell migration by affecting the transport of water to cell protrusions formed during migration and that it is, therefore, a potential therapeutic target to treat tumours (Verkman, 2005; Hu, 2006; Papadopoulos, 2008). It was also reported that AQP-1 was strongly expressed in endothelial cells of the capillary and postcapillary venules in Lewis-lung-carcinoma tumour tissues in mice, which could be inhibited by the mild diuretic Acetazolamide (Xiang, 2004).

In summary, our results showed progressive changes in the pituitary gland upon DES treatment. Anatomically, the vault-shape enlargement of the pituitary gland and the discontinuous CSF signal in the mid-sagittal section evident upon MRI strongly suggested that hypophyseal proliferation and angiogenesis resulted from DES treatment. Non-endothelial hypophyseal VEGF expression appears to function during angiogenic initiation, whereas subsequent sustained angiogenesis may partially rely on the endothelial expression of AQP-1. Disrupting the action of VEGF and AQP-1 depending on the stage of angiogenesis may provide an important therapeutic strategy to control tumour cell growth, both in vitro and clinically.

\section{References}

Arnaoutova I, Cawley NX, Patel N, Kim T, Rathod T, Loh YP. Aquaporin 1 is Important for Maintaining Secretory Granule Biogenesis in Endocrine Cells. Mol Endocrinol 2008;22:192434.

Banerjeei SK, Zoubine MN, Sarkar J. 22Methoxyestradiol blocks estrogen induced rat pituitary tumor growth and tumor angiogenesis possible role of vascular endothelial growth factor. Anticacer Res 2000;20:2641-5.

Bochner BH, Cote RJ, Weidner N. Angiogenesis in bladder cancer: relationship between microvessel density and tumour prognosis. $J$ Natl Cancer Inst 1995;87:1603-12.

Cracchilolo D, Swick JW, McKiernan L. Estrogen-dependent gfowth of rat pituitary tumor involves, but does not require, a high level of vascular endothelial growth factor. Exp Biol Med 2002;227:492-9.

Dolman D, Drndarski S, Abbott NJ, Rattray M. Induction of aquaporin 1 but not aquaporin 4 messenger RNA in rat primary brain microvessel endothelial cells in culture. J Neurochem 2005;93: 825-33.

Folkman J. What is the evidence that tumours are angiogenesis dependent? J Nat Cancer Inst 1990;82:4-6.

Gimbrone Jr MA, Leapman S, Cotran RS. Tumour dormancyin vivo by prevention of neovascularisation. J Exp Med 1972;73:46173.

Gimbrone Jr MA, Leapman S, Cotran RS. Tumour angiogenesis: iris neovascularisation at a distance from intraocular tumours. J Natl Acad Inst 1973;50:219-28.

Gospodarowicz D, Lau K. Pituitary follicular cells secret a novel heparin binding growth factor specific for vascular endothelial cells. Biochem Biophys Commun Res 1989;161:851.

Goth MI, Hubona E, Raptis S. Physiological and pathological angiogenesis in the endocrine system. Microsc Res Tech 2003;60:98106.

Guinebretiere JM. Angiogenesis and breast neoplasms. The pathologist's point of view. Gynecol Obstet Fertil 2005;33:140-6.

Heaney AP, Fernando M, Melmed S. Functional role of estrogen in pituitary tumor pathogenesis. J Clin Invest 2002;109:277-83.

$\mathrm{Hu} \mathrm{J}$, Verkman AS. Increased migration and metastatic potential of tumor cells expressing aquaporin water channels. FASEB J 2006; 20:1892-4.

Mukdsi JH, De Paul AL, Gutiérrez S, Roth FD, Aoki A, Torres AI. Subcellular localisation of VEGF in different pituitary cells. Changes of its expression in oestrogen induced prolactinomas. J Mol Histol 2005; 36:447-54.

Kuwahara S, Maeda S, Tanaka, Hayakawa T, Seki M. Expression of Aquaporin Water Channels in the Rat Pituitary Gland. J Vet Med Sci 2007;69:1175-8.

Loyhrer P, Gloddek J,Hopfner U. Vascular endothelial growth factor production and regulation in rat and human pituitary tumor cells in vitro. Neuroendocrinol 2001;74:95-105.

Maeda K, Chung Y-S, Takasuka S. Tumour angiogenesis as predictor of recurrence in gastric carcinoma. J Clin Oncol 1995;13: 47781.

McCabe CJ, Boelaert K, Tannahill LA. Vascular endothelial growth fac tor, its receptor KDRPFIk2l, and pituitary tumor transforming gene in pituitary tumors. J Clin Endocrinol Metab 2002; 87:4238-44

Pan $L$, Liu $Y$, Chen Z. Associatio $n$ of vascular endothelial growth factor expression with intratumoral microv essel density and cavernous sinus invasion in pituitary adenomas. Chin J Neurosurg 2003;19: 285-7.

Papadopoulos MC, Saadoun S, Verkman AS. Aquaporins and cell migration. Pflugers Arch 2008;456:693-700.

Rudin M, Briner $U$, Doepfner W. Quantitative magnetic resonance imaging of estradiol-induced pituitary hyperplasia in rats. Magn Reson Med 1988;7:285-91.

Satoh $\mathrm{H}$, Kajimura T, Chen CJ. Invasive pituitary tumors in female F344 rats induced by estradiol dipropionate. Toxicol Pathol $1997 ; 25: 462-9$. 
van Nesselrooij JH, Szeverenyi NM, Ritter-Hrncirik C. Rat pituitary changes observed with magnetic resonance imaging following removal of estrogen stimulus: correlation with histopathology and immunohistology. Carcinogenesis 1992;13:277-82.

Verkman AS. More than just water channels: unexpected cellular roles of aquaporins. J Cell Sci 2005;118:3225-32.

Warth A, Mittelbronn M, Hülper $P$, et al. Expression of the water channel protein aquaporin-9 in malignant brain tumors. Appl Immunohistochem. Mol Morphol 2007;15:193-8.

Weidner N, Semple JP, Welch WR, Folkman J. Tumour angiogenesis and metastasis: correlation in invasive breast carcinoma. N Engl
J Med 1991;324:1-8.

Weidner N, Folkman J, Pozza F. Tumour angiogenesis: a new significant and independent prognostic indicator in early stage breast carcinoma. J Natl Cancer Inst 1992;84:1875-87.

Xiang Y, Ma B, Li T, Gao JW, Yu HM, Li XJ Acetazolamide inhibits aquaporin-1 protein expression and angiogenesis. Acta Pharmacol Sin 2004;25:812-6.

Zhao W, Yuan F, Li G, et al. Long-term application of diethylstilbestrol upregulates expressions of $\mu$ - and $m$-calpains in pituitary intermediate lobe of female Wistar rats. Neural Regener Res $2007 ; 2: 276-80$. 